\title{
A Study of Performance on SMP and Distributed Memory Architectures Using A Shared Memory Programming Model
}

\author{
Eugene D. Brooks, III \\ Karen H. Warren \\ This paper was prepared for submittal to \\ Supercomputing 97 \\ San Jose, CA \\ November 15 - 21, 1997
}

August 8, 1997

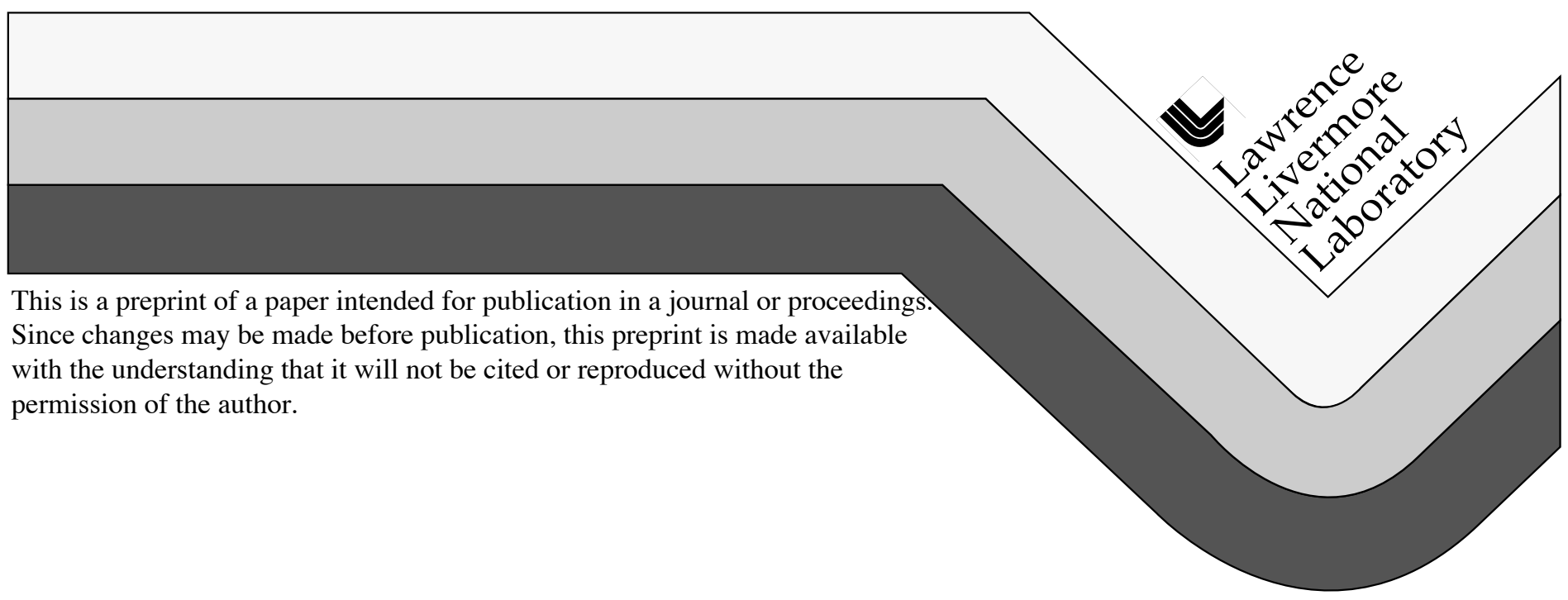




\section{DISCLAIMER}

This document was prepared as an account of work sponsored by an agency of the United States Government. Neither the United States Government nor the University of California nor any of their employees, makes any warranty, express or implied, or assumes any legal liability or responsibility for the accuracy, completeness, or usefulness of any information, apparatus, product, or process

disclosed, or represents that its use would not infringe privately owned rights. Reference herein to any specific commercial product, process, or service by trade name, trademark, manufacturer, or otherwise, does not necessarily constitute or imply its endorsement, recommendation, or favoring by the United States Government or the University of California. The views and opinions of authors expressed herein do not necessarily state or reflect those of the United States Government or the University of California, and shall not be used for advertising or product endorsement purposes. 


\title{
A Study of Performance on SMP and Distributed Memory Architectures Using a Shared Memory Programming Model *
}

\author{
Eugene D. Brooks III and Karen H. Warren \\ University of California \\ Lawrence Livermore National Laboratory \\ Livermore, California 94550 \\ brooks3ellnl.gov kwarren@llnl.gov
}

\begin{abstract}
:
In this paper we examine the use of a shared memory programming model to address the problem of portability of application codes between distributed memory and shared memory architectures. We do this with an extension of the Parallel C Preprocessor. The extension, borrowed from Split-C and AC, uses type qualifiers instead of storage class modifiers to declare variables that are shared among processors. The type qualifier declaration supports an abstract shared memory facility on distributed memory machines while making direct use of hardware support on shared memory architectures.

Our benchmarking study spans a wide range of shared memory and distributed memory platforms. Benchmarks include Gaussian elimination with backsubstitution, a two-dimensional fast Fourier transform, and a matrix-matrix multiply. We find that the type-qualifier-based shared memory programming model is capable of efficiently spanning both distributed memory and shared memory architectures. Although the resulting shared memory programming model is portable, it does not remove the need to arrange for overlapped or blocked remote memory references on platforms that require these tuning measures in order to obtain good performance.
\end{abstract}

\section{Keywords:}

Parallel C, type qualifier, shared memory, distributed memory

\section{Introduction}

Message passing has evolved as the portability vehicle of choice for parallel programs that must run on both distributed memory and shared memory architectures. This has occurred because message passing is relatively easy to implement. Widely accepted standards such as the Message Passing Interface (MPI) [1] exist with implementations available on many shared and distributed memory platforms. The use of message passing 
is a natural choice on distributed memory platforms, but its use on shared memory systems can sacrifice performance in applications that are sensitive to communication latency and bandwidth. Whether one can devise alternative parallel programming paradigms that efficiently span shared and distributed memory architectures in a portable manner is the subject of this paper.

We investigate a shared memory paradigm as a portability vehicle for programs that must run on both shared and distributed memory architectures. The goal of the paradigm is to make the low latency communication capability of a shared memory platform available to the programmer while preserving portability to a distributed memory platform. Applications written in such a programming model have a communication granularity that is driven by the characteristics of the algorithm itself instead of being strongly influenced by the choice of programming model. If an algorithm's dependence upon fine-grained communication is unavoidable, one will find that shared memory platforms provide the best execution vehicle. If, on the other hand, the application can be coded in a way that makes efficient use of course-grained communication, distributed memory platforms will provide acceptable performance.

The concept that makes it possible to target both shared memory and distributed memory platforms using a shared memory programming model is the treatment of data sharing keywords as type qualifiers. This idea was introduced in the Split-C [2] and AC [3] compilers that target distributed memory parallel computers. In Split-C, data sharing keywords interpreted as type qualifiers enable the translation of abstract shared memory references into Active Message [4] calls that effect communication between processors. In $\mathrm{AC}$, a similar type qualifier strategy is used to translate shared memory references into direct use of the Cray T3D and T3E remote memory reference hardware support, after address computation has been handled in software. These compiler efforts left the issue of portability to shared memory computing platforms untested.

In our study, we evaluate the type qualifier approach to shared memory declarations as an extension [5] of the Parallel C Preprocessor (PCP) [6], a parallel extension of the C programming language originally developed for shared memory multiprocessing platforms. Our implementation is a source-to-source translator that takes full advantage of the fact that type qualifier shared memory declarations can be efficiently translated to direct use of the hardware address calculation and memory sharing mechanisms on a shared memory computing platform. The result is a shared memory programming model that is portable across a wide range of distributed memory and shared memory platforms and does not impose any gratuitous software overheads on any given architectural target.

Although the type qualifier approach to data sharing keywords can lead to an efficient implementation of interprocessor communication on a wide variety of machine 
architectures, it does not change the fundamental requirements of exploiting latency hiding mechanisms on machines such as the Cray T3D or T3E, and avoiding short messages on machines such as the Meiko CS-2. In the case of the Cray T3D and T3E, the traditional notion of vector processing applied to the movement of data between shared and private memory is effective in hiding communication latency. On the Meiko CS-2, one must resort to blocked data transfer to obtain good performance.

The paper is organized as follows: we first describe the relevant aspects of the extended PCP programming model. We then describe the target architectures, the PCP translations, and library support. Then we present the performance results for a Gaussian elimination, a 2-D fast Fourier transform, and a matrix-matrix product benchmark. We end with a discussion.

\section{Shared Memory Model}

On conventional shared memory multiprocessing platforms, shared memory is supported in hardware. This is done in a way that is transparent to the expression recognition and evaluation mechanisms in a compiler. A data object is placed in a shared memory segment by a declaration that modifies the storage class of the object, hence the term storage class modifier, instructing the compiler to pass the sharing status through to the assembler and linker. As an example, consider below the declaration for foo, a shared static integer in which the keyword shared modifies the storage class static for the variable.

shared static int foo;

With the storage class modifier interpretation of data sharing keywords, a pointer to an object is generic. The sharing status of the object referred to is handled by the hardware memory management unit in the processor.

One can extend this approach to distributed memory platforms by intercepting shared addresses before loads and stores are issued. However, an alternative approach for declarations that offers more expressive power to the programmer is possible. This approach to shared objects interprets data sharing keywords as type qualifiers. The keyword shared affects the type and not the storage class of an object. Our example of the declaration for foo is now written

static shared int foo;

which appears to be a trivial syntactic change. The adjustment, however, opens up an entirely new range of declarations. Consider the following pointer declaration: 
shared int * shared * private bar;

The variable bar itself resides in private memory. It refers to a pointer residing in shared memory which finally refers to an integer residing in shared memory. The type qualifier interpretation of data sharing keywords provides the programmer with the ability to indicate sharing status at all levels of indirection. It also provides the compiler with the needed hooks to handle shared memory references on a distributed memory platform.

Unlike the Split-C and AC compilers that are based on the GNU C compiler and produce assembly language, we have implemented our extension of the PCP programming model as a source-to-source translator. The backend target is the vendor-supported C compiler combined with a runtime library that handles synchronization and communication operations that are not part of the architectural support provided by the vendor.

Implementing PCP as a source-to-source translator creates a more easily ported compiler and leverages the substantial effort vendors usually make to optimize the performance of their proprietary $\mathrm{C}$ compilers.

\section{Target Architectures}

In this paper we evaluate the utility of the type qualifier approach to shared memory declarations on the following platforms: the DEC 8400 symmetric shared memory multiprocessor, the SGI Origin $2000^{1}$ coherent cache non-uniform memory access architecture, the Cray T3D and $\mathrm{T}^{2} \mathrm{E}^{2}$ distributed memory platforms which support remote memory references in hardware, and the Meiko CS-2 which supports one-sided messages in software.

\section{DEC 8400}

The DEC Alpha 8400 symmetric shared memory multiprocessor is a conventional bus-based parallel system. The DEC platform may have up to 12 processors that share a system bus possessing a sustainable bandwidth of 1600 megabytes per second. The memory system can support up to 16-way interleaving, the actual amount of memory interleave depending upon the specific memory configuration. Further details can be found in the Technical Summary [7] for the DEC 8400 system. For our benchmarking we use a DEC 8400 with 8 processors running at 440 megahertz with 4-way interleaved memory.

The DEC Alpha processor provides a weakly consistent memory model [8]. If program correctness requires the ordering of memory operations, a memory barrier instruction is used to force pending memory operations to complete before further instructions are allowed to issue. Load-linked and store-conditional instructions are provided in the DEC 
Alpha processor to support synchronization operations.

\section{SGI Origin 2000}

The SGI Origin 2000 provides a shared memory architecture implemented as a distributed shared memory system as opposed to a symmetric bus architecture. The Origin 2000 system [9] is built from a number of nodes, each containing two R10000 processors and their associated second level caches. A portion of the system memory and the cache directory are positioned on the node as well. The nodes are interconnected by a communications fabric implementing a hypercube for modest configurations of up to 32 nodes. A directory-based cache coherence protocol is implemented over this fabric in order to provide a system-wide cache coherent shared memory.

The SGI Origin 2000 offers increased scalability because it does not depend upon a single system bus to provide for shared memory. Unlike the case for a symmetric shared memory platform, however, application performance can be sensitive to data layout. Data layout is controlled on the Origin 2000 by influencing the page placement algorithms of the virtual memory system at runtime. The memory model provided on the Origin 2000 is sequentially consistent [10]. Load-linked and store-conditional instructions are provided to support synchronization.

\section{Cray T3D and T3E}

The Cray T3D and T3E platforms are distributed memory machines that employ DEC Alpha microprocessors and a torus network to implement interprocessor communication. Each processor operates on its own local address space but mechanisms are provided to support remote memory references through the communications network. In the T3D, remote memory references are implemented in the support circuits surrounding the processor. Before a load or store is issued by the processor, a special instruction may be used to set the target CPU to a non-local processor.

A prefetch queue and a block transfer engine permit the programmer to hide latency on the Cray T3D. We employ the prefetch queue to implement vector fetches from distributed to local memory. A remote read-modify-write cycle and a hardware barrier instruction are provided to support synchronization. To obtain the best possible performance, a large fraction of the PCP remote memory reference runtime library support is written in assembly language on the T3D.

The multiprocessing support architecture introduced in the T3D has been substantially refined in the Cray T3E. Memory mapped registers, called E registers, are used to access all of the multiprocessing support operations. Through the use of the E register mechanism remote memory references, read-modify-write operations, and a barrier 
instruction can be accessed. E register operations can also be used to implement efficient vector transfers between local and distributed memory. A key advantage of the T3E is that the E register mechanism is directly accessible from an optimizing $\mathrm{C}$ compiler.

Both the Cray T3D and T3E provide a weakly consistent memory model. This occurs at two levels. The DEC Alpha processor, used in both platforms, implements weakly ordered memory operations. The remote memory reference operations implemented in the multiprocessing support logic are also weakly ordered. One must wait on remote reads to complete in order to ensure that they precede other remote memory references. Remote memory writes are tracked in the support logic and their completion is explicitly waited for if algorithm correctness depends upon memory ordering.

\section{Meiko CS-2}

The Meiko CS-2 is a distributed memory computing platform based on the SUN SPARC processor, using a separate Elan [11] processor to implement interprocessor communication across a network. The Elan processor on the local node executes a communications protocol that communicates with the Elan processor on a remote node to implement memory-to-memory (DMA) transfers. Since the communication protocol runs in software on the Elan, the startup latency for data transfers is significant. This requires data movement to occur in large block transfers in order to obtain good performance.

The memory-to-memory transfers implemented by the Elan communications processor on the Meiko CS-2 are weakly ordered. One must explicitly wait on an event associated with a DMA operation to ensure that it is complete before continuing with further data transfers if algorithm correctness depends upon ordering. There are no remote read-modify-write cycles implemented in the Elan library. As a result, we were forced to resort to Lamport's algorithm [12] for mutual exclusion.

\section{PCP Translations and Runtime Libraries}

The PCP programming model is implemented as a source-to-source translator that performs a full parse of the PCP programming language. It produces ANSI C augmented by either calls to communication and synchronization routines in the PCP runtime library or vendor-specific functionality. The translator is implemented as a single program with several output options selected by a flag. One or more output options may be viable on a given target machine and a runtime library is generally associated with each output option.

\section{Shared Memory Platforms}


Some vendors of shared memory multiprocessor systems do not provide shared static variables in their C compiler that we can target with our PCP translator. We have used two strategies to make up for this lack of direct support for shared variables. In the first strategy we convert static addresses to shared status in place. In the second approach we create a shared duplicate of the program data area at an offset address.

\section{Conversion in Place}

Some shared memory multiprocessor systems provide system calls that are capable of remapping an existing valid region of virtual memory. If the address ordering of variables defined in a source file is preserved by the loading process, we can arrange to convert the variables to shared status in place. To do this, the PCP translator produces two backend files for each PCP source file. One file contains the code, private data definitions and externalized declarations for the shared variables. The second file contains the data definitions for the shared variables.

When the parallel program is built, all of the data definitions for shared variables are concatenated into a single file along with a header and trailer that mark the beginning and end of the shared memory region. When the resulting binary program is run, a page aligned region of memory is found that starts in the header and ends in the trailer. This data region, which contains all of the static shared variables, is then written out to a file and mapped back in to give it shared semantics under process fork. Once the shared data segment is created, the PCP runtime forks the required number of processes to start the parallel job.

In addition to the above tasks of creating the static shared data segment and starting the parallel job, the PCP runtime library implements locks for critical regions, dynamic allocation of shared memory, and barrier synchronization.

\section{Address Offsetting}

On platforms that do not preserve address ordering for variables defined in a single source file, or are not capable of converting an existing virtual memory region to shared status, we have implemented a second strategy for creating the shared data segment. In this case, the PCP translator adds a constant offset to the address of all shared static variables in order to reach an unused portion of virtual memory. Before parallel processes are forked, a shared copy of the entire program data area is created at this offset address.

The address offsetting strategy of establishing the shared data segment simplifies building a code and makes library management easier. The cost to be paid for this convenience is the additional runtime overhead of adding a constant offset to the addresses of staticly scoped shared variables. In our benchmarks, which go to some 
degree of effort to minimize shared memory use, this additional overhead has amounted to only a few percent.

Other than the different way of establishing the shared memory segment and the offset added to static shared addresses by the PCP translator, the PCP runtime support is identical to that of conversion in place described above. On some platforms, both options can be supported.

\section{Distributed Memory Platforms}

For distributed memory platforms the PCP translator manages the distribution of arrays across the processors. Arrays are distributed on object boundaries in such a manner that the first element of a staticly allocated array resides on processor zero. Pointers to shared objects may refer to any array element in the same unrestricted manner as conventional pointers in C. Pointer arithmetic and remote memory reference operations are handled in software if required for a given architecture.

The format of a pointer to a shared object depends upon the target architecture. Some platforms implement pointers that are 64 bits wide and admit the packing of the processor index into unused address bits. An example of this is the Cray T3D which leaves the upper 16 bits of a pointer value unused. A processor index for up to $64 \mathrm{~K}$ processors can be accommodated in this unused field. On other platforms a pointer is only 32 bits wide and will not accommodate the available system-wide memory for even a modest number of processors. In this case, we define a pointer to a shared object as a structure that contains the address and processor index as separate fields. Using a structure value for pointers is cleaner, but most $\mathrm{C}$ compilers are clumsy when dealing with structure values as arguments to or returned from subroutines.

In the PCP implementation for distributed memory platforms, the programmer provides information about the processor count at compile time. A shared array of size $\mathrm{N}$ is allocated (N+NPROCS-1)/NPROCS elements in the C language output for the array definition, where NPROCS is the lower bound on the number of processors that will be used to execute the application.

All distributed memory implementations require an implementation of arithmetic for pointers to shared data. These routines are inlined if the backend compiler on the target platform supports procedure inlining. Routines that support remote references for all of the ANSI C basic data types, and aggregate types such as structures, must also be written for the target architecture. The only restriction that this places on the target architecture is that one-sided communication be supported. In addition to functions to perform address arithmetic and remote memory operations, library support for parallel job startup, 
allocation of distributed arrays, mutual exclusion, and barrier synchronization must be written for the target architecture.

If possible, vendor-specific functionality is employed to optimize the performance of communication and synchronization operations. On the Cray T3D, remote memory reference routines are written in assembly language that directly manipulates the hardware communications support. Similarly, on the Cray T3E, remote memory reference operations are implemented as inlined functions that directly access the $\mathrm{E}$ register mechanism. This removes routine overhead from single word remote memory accesses. On the Meiko CS-2, the Elan one-sided communications library [13] is used to implement remote memory references. There is substantial software overhead associated with the Elan library.

Each of the distributed memory target architectures offers means to reduce the impact of communication latency. This can be exploited in PCP by using blocked data movement, implemented as remote access to $\mathrm{C}$ structures, or vector data movement, implemented with a subroutine interface. On the Meiko CS-2, the movement of large blocks of data can amortize the software costs of communication startup. The hardware prefetch queue on the T3D is used to efficiently overlap the fetch of individual words in a C structure, and to efficiently overlap the fetch of single word array elements distributed across the processors. Similarly, the E register mechanism is used on the T3E to overlap remote memory access.

\section{Benchmark Results}

In order to evaluate the utility of the type qualifier shared memory declarations, we have implemented Gaussian elimination with backsubstitution, a 2-D fast Fourier transform, and a matrix-matrix multiply in the extended PCP programming model. The performance of these benchmarks was evaluated on all of the target architectures.

\section{Gaussian Elimination}

Our first benchmark is a parallel version of the Gaussian elimination algorithm [14]. For a dense linear system of size $\mathrm{N} \times \mathrm{N}$ the algorithm begins with $\mathrm{N}$ reduction steps wherein scalar multiples of the ith row, known as the pivot row, are subtracted from the rows below in order to reduce the matrix to upper triangular form. Once the reduction is complete, backsubstitution produces the solution vector elements in succession.

In the parallel version, an array of flags located in shared memory indicates when a pivot row is ready for use in the reduction. The same array of flags, being reset to zero, indicates when an element of the solution vector is ready for use in the backsubstitution. 
At the start of the algorithm a processor's share of the rows of the matrix, and the associated portion of the right hand side, are copied from shared memory to private memory. This copying is carried out element-by-element, but may be handled in a routine that executes the copy in a vectorized manner if element-by-element communication can be overlapped on a given architecture. A pivot row, and/or an element of the solution vector, is copied back out to shared memory when the data is ready for use by other processors. We note that the ordering relationship between the setting of a flag and the assignment of its corresponding data must be carefully enforced on machines for which the memory consistency model is not sequential.

In presenting these performance results, we report the rate at which the parallel code executes a $1024 \times 1024$ linear system solve, and the speedup compared to the parallel code executed by one processor, as a function of the number of processors. All rates are expressed in millions of floating point operations per second (MFLOPS). Execution times are measured on dedicated or suitably gang scheduled [15] machines. To provide a point of reference, we also report the rate at which a processor can repetitively add a scalar multiple of a vector to another vector (DAXPY). We use a vector length of 1000 so all operations hit cache. In Tables 1 though 5, the floating point rate for parallel execution of the Gaussian elimination benchmark as a function of the processor count, $\mathrm{P}$, is shown in the column labeled MFLOPS. The speedup, measured as the execution time of the parallel code running with one processor divided by the time for P processors, is shown in the column labeled Speedup.

In Table 1 we show the performance of an 8 processor DEC 8400 system on the Gaussian elimination benchmark as a function of the number of processors. The basic DAXPY speed of a processor on this machine is 157.9 MFLOPS for compiled C code. The speedups in the table are superlinear. This is caused by the increasing amount of high speed cache memory available as the processor count is increased. The MFLOP rate does not exceed the number of processors times the cache hit DAXPY rate, as one would expect. 


\begin{tabular}{|r|r||r|}
\hline $\mathrm{P}$ & MFLOPS & Speedup \\
\hline \hline 1 & 41.66 & 1.00 \\
\hline \hline 2 & 168.26 & 4.04 \\
\hline \hline 3 & 272.63 & 6.54 \\
\hline \hline 4 & 365.05 & 8.76 \\
\hline \hline 5 & 448.70 & 10.77 \\
\hline 6 & 531.80 & 12.77 \\
\hline 77 & 606.70 & 14.56 \\
\hline \hline 8 & 642.92 & 15.43 \\
\hline
\end{tabular}

\section{Table 1: Gaussian Elimination Performance on the DEC 8400}

In Table 2 we show the results for the Gaussian elimination benchmark on the SGI Origin 2000 platform. The basic DAXPY speed for a single processor of the Origin 2000 is 96.62 MFLOPS. Speedups are superlinear for processor counts past 20, again due to the scaling of cache size with processor count.

\begin{tabular}{|r|r||r|}
\hline $\mathrm{P}$ & MFLOPS & Speedup \\
\hline \hline 1 & 55.35 & 1.00 \\
\hline \hline 2 & 135.71 & 2.45 \\
\hline \hline 4 & 267.88 & 4.84 \\
\hline \hline 8 & 539.79 & 9.75 \\
\hline 16 & 997.12 & 18.01 \\
\hline 20 & 1139.56 & 20.59 \\
\hline \hline 25 & 1380.62 & 24.94 \\
\hline \hline 30 & 1495.68 & 27.02 \\
\hline
\end{tabular}

Table 2. Gaussian Elimination Performance on the SGI Origin 2000

In Table 3 we show the results for the Gaussian elimination benchmark on the Cray T3D platform. Due to the capability of the T3D to overlap remote memory accesses, using a 
vectorized interface to the communication hardware can reduce the overheads associated with communication. The columns labeled MFLOPS and Speedup are for scalar implementations of shared memory access. The performance of the benchmark using vector access to shared memory is shown in the column labeled MFLOPS Vector, and the associated speedup is shown in the column labeled Speedup Vector. The use of overlapped communication improves both absolute performance and speedup significantly. The basic DAXPY speed for a single processor of the T3D is 11.86 MFLOPS.

\begin{tabular}{|r||r||r||r||r|}
\hline $\mathrm{P}$ & MFLOPS & Speedup & MFLOPS Vector & Speedup Vector \\
\hline \hline 1 & 8.37 & 1.00 & 10.10 & 1.00 \\
\hline \hline 2 & 15.99 & 1.91 & 20.05 & 1.99 \\
\hline \hline 4 & 30.33 & 3.62 & 39.83 & 3.94 \\
\hline \hline 8 & 52.63 & 6.29 & 79.21 & 7.84 \\
\hline \hline 16 & 78.22 & 9.35 & 143.62 & 14.22 \\
\hline 32 & 94.44 & 11.28 & 277.63 & 27.49 \\
\hline
\end{tabular}

\section{Table 3. Gaussian Elimination Performance on the Cray T3D}

In Table 4 we show the results for the Gaussian elimination benchmark on the Cray T3E platform. As was the case for the T3D, the performance for both scalar and vector access to shared memory is shown. Vector access to shared memory is required to obtain good performance on the T3E. The basic DAXPY speed for a single processor of the T3E is 29.02 MFLOPS.

\begin{tabular}{|r||r||r||r||r|}
\hline $\mathrm{P}$ & MFLOPS & Speedup & MFLOPS Vector & Speedup Vector \\
\hline \hline 1 & 17.91 & 1.00 & 18.51 & 1.00 \\
\hline \hline 2 & 35.58 & 1.99 & 37.27 & 2.01 \\
\hline \hline 4 & 65.04 & 3.63 & 73.57 & 3.97 \\
\hline \hline 8 & 112.83 & 6.30 & 145.06 & 7.84 \\
\hline \hline 16 & 182.02 & 10.16 & 289.31 & 15.63 \\
\hline 32 & 247.63 & 13.83 & 558.66 & 30.18 \\
\hline
\end{tabular}

Table 4. Gaussian Elimination Performance on the Cray T3E-600 
In Table 5 we show the results for the Gaussian elimination benchmark on the Meiko CS-2. The basic DAXPY speed for a single processor on the CS-2 is 14.93 MFLOPS. Although the Meiko Elan library provides for overlapped communication, attempting to overlap small one-sided messages does not result in any performance gain. The substantial latency placed on single word communication prevents this particular algorithm for Gaussian elimination from performing well on the Meiko CS-2. Performance could be improved by changing the data layout so that a given row of the matrix is contained on one processor, enabling more efficient use of the DMA capability on the CS-2, and by using a software tree to broadcast pivot rows.

\begin{tabular}{|r||r|r|}
\hline $\mathrm{P}$ & MFLOPS & Speedup \\
\hline \hline 1 & 3.79 & 1.00 \\
\hline 2 & 6.15 & 1.62 \\
\hline \hline 3 & 8.16 & 2.15 \\
\hline \hline 4 & 9.81 & 2.59 \\
\hline \hline 5 & 11.14 & 2.94 \\
\hline \hline 8 & 13.92 & 3.67 \\
\hline \hline 16 & 14.01 & 3.70 \\
\hline
\end{tabular}

\section{Table 5: Gaussian Elimination Performance on the Meiko CS-2}

\section{Fast Fourier Transform}

The FFT benchmark is a fast Fourier transform (FFT) of a $2048 \times 2048$ array of complex values composed of 32 bit floating point data. The 2-D FFT is executed as 2048 independent 1-D Fourier transforms in the $x$ direction, followed by a similar set of 1-D transforms running in the $y$ direction. For the sake of portability, we use compiled $\mathrm{C}$ code [16] for the 1-D transform for all target platforms.

The parallel version of the 2-D transform is straightforward. The 2048 independent 1-D transforms in the $x$ direction are shared by the participating processors, each copying the 1-D stripe to private memory where the 1-D transform is computed, and then copying the data back out to shared memory. A barrier separates the computation of the sweep in the $x$ direction from the sweep in the $y$ direction. The work of the sweep in the $y$ direction is then shared by the processors followed by a barrier before the 2-D FFT routine returns.

The work load is balanced for any power of two for the processor count. The shared data 
accesses can be vectorized with a stride of one for the sweeps in the $y$ direction and with a stride of 2048 for the sweeps in the $x$ direction. On coherent cache based shared memory multiprocessors, the stride of 2048 can be unfortunate as will be shown in the benchmarks. This is dealt with by padding the arrays by one element. The index scheduling for the sweeps in the $x$ direction can also be unfortunate on coherent cache based shared memory multiprocessors, leading to false sharing of cache lines. This is dealt with by blocking the index scheduling.

In presenting performance results, we document the time in seconds for computing a $2048 \times 20482$-D transform with serial code, and the execution time and speedup of the parallel code as a function of processor count. The speedup is relative to the time for one processor executing the parallel code. The efficiency relative to the serial algorithm is known given the separate timing of the serial code that we provide.

In Table 6 we show the performance of the DEC 8400 platform on the FFT benchmark. The execution time and associated speedup, as a function of processor count, $\mathrm{P}$, are shown in the columns labeled Time and Speedup, respectively. In the columns labeled Time Blocked and Speedup Blocked, we show the execution time and associated speedup for a version of the FFT benchmark that blocks the index scheduling in a manner that minimizes false sharing. In the columns labeled Time Padded and Speedup Padded, we show the execution time and associated speedup for the blocked version of the FFT benchmark that pads the arrays to minimize cache line collisions.

The execution time for a serial implementation of a $2048 \times 2048$ FFT is 10.82 seconds. The execution time for the same serial 2-D transform with the arrays padded by an extra element to reduce cache line collisions when reading or writing memory in the $x$ direction, is 8.55 seconds. Within measurement error, the timings for the serial code are identical to the timings for the associated parallel code executed with one processor. This indicates that parallelization overhead is insignificant. We note that blocked index scheduling does not change performance in a significant way on the DEC 8400. Since we know that false sharing of cache lines must be occurring on the $x$ directional sweep, the cost of this communication between processors must be relatively low. The best absolute performance and speedup is obtained by padding the arrays to avoid cache line collisions. 


\begin{tabular}{|l||r||r||r||r||r||r|}
\hline $\mathrm{P}$ & Time & Speedup & Time Blocked & Speedup Blocked & Time Padded & Speedup Padded \\
\hline \hline 1 & 10.75 & 1.00 & 10.75 & 1.00 & 8.55 & 1.00 \\
\hline \hline 2 & 5.85 & 1.84 & 5.48 & 1.96 & 4.30 & 1.99 \\
\hline \hline 4 & 2.97 & 3.62 & 2.93 & 3.67 & 2.18 & 3.92 \\
\hline \hline 8 & 1.82 & 5.91 & 1.90 & 5.66 & 1.15 & 7.43 \\
\hline
\end{tabular}

\section{Table 6. FFT Performance on the DEC 8400}

The SGI Origin 2000 is a distributed shared memory platform wherein each page resides on a computational node. If one processor performs the initialization of the 2-D array, all of the pages of memory reside on the node that contains this processor, leading to a performance bottleneck. Obtaining good performance required adjustments to the code to deal with this problem. We also found that the presence of a bottleneck in the virtual memory system prevented good performance on an initial 2-D FFT when processors were taking page or memory management unit faults. This was addressed by performing the FFT twice and timing the second instance.

In Table 7 we show the performance of the SGI Origin 2000 platform on the FFT benchmark. In the columns labeled Time Sinit and Speedup Sinit we show the execution times and relative speedup as a function of processor count, $\mathrm{P}$, for a version of the FFT benchmark wherein a single processor does the initialization. As a result, pages are located on one node. In the columns labeled Time Pinit and Speedup Pinit we show the execution times and relative speedup for a version of the FFT benchmark in which the processors share the initialization tasks. Pages are therefore distributed across the machine. The improvement in performance obtained by distributing the pages across the computational nodes is clear. All further benchmark measurements on the SGI Origin 2000 use a parallel initialization to remove this potential bottleneck.

In the columns labeled Time Blocked and Speedup Blocked we show the execution times and relative speedup for a version of the FFT benchmark in which index scheduling in the $x$ direction is blocked to reduce false sharing. A significant increase in speedup is obtained. Finally, in the columns labeled Time Padded and Speedup Padded, we show the execution times and relative speedup for a blocked version of the FFT benchmark in which the $y$ array dimension is padded by one element to reduce cache line collisions. As was the case for the DEC 8400, the best absolute performance and relative speedups are obtained by padding the arrays.

The execution time for a serial implementation of a $2048 \times 2048$ FFT is 11.0 seconds. 
The execution time for the same 2-D transform with the arrays padded by an extra element to avoid cache line collisions, is 7.58 seconds. Comparing the serial implementation execution times with that of the parallel implementation using one processor, we see that the parallelization overhead is low.

\begin{tabular}{|c|c|c|c|c|c|c|c|c|}
\hline $\mathrm{P}$ & $\begin{array}{l}\text { Time } \\
\text { Sinit }\end{array}$ & $\begin{array}{l}\text { Speedup } \\
\text { Sinit }\end{array}$ & $\begin{array}{l}\text { Time } \\
\text { Pinit }\end{array}$ & $\begin{array}{l}\text { Speedup } \\
\text { Pinit }\end{array}$ & $\begin{array}{c}\text { Time } \\
\text { Blocked }\end{array}$ & $\begin{array}{l}\text { Speedup } \\
\text { Blocked }\end{array}$ & $\begin{array}{c}\text { Time } \\
\text { Padded }\end{array}$ & $\begin{array}{l}\text { Speedup } \\
\text { Padded }\end{array}$ \\
\hline 1 & 11.03 & 1.00 & 11.08 & 1.00 & 11.20 & 1.00 & 7.64 & 1.00 \\
\hline 2 & 7.44 & 1.48 & 7.44 & 1.49 & 6.23 & 1.80 & 3.85 & 1.98 \\
\hline 4 & 4.50 & 2.45 & 4.32 & 2.56 & 3.57 & 3.14 & 1.97 & 3.88 \\
\hline 8 & 3.09 & 3.57 & 2.61 & 4.25 & 2.02 & 5.54 & 1.03 & 7.42 \\
\hline 16 & 2.68 & 4.12 & 1.44 & 7.75 & 1.10 & 10.18 & 0.54 & 14.15 \\
\hline
\end{tabular}

\section{Table 7. FFT Performance on the SGI Origin 2000}

In Table 8 we show the performance of the Cray T3D platform on the FFT benchmark. The execution time and speedup for a version of the FFT that uses scalar access to shared memory is in the columns labeled Time and Speedup, respectively. The execution time and speedup for a version of the FFT that uses vector access to shared memory is shown in the columns labeled Time Vector and Speedup Vector, respectively. The execution time for a serial implementation of a $2048 \times 2048$ FFT is 44.18 seconds. On the T3D, the use of vector access to shared memory produces both the best absolute performance and relative speedup. The speedup of 251 for 256 processors demonstrates the scalability of the PCP implementation of the FFT benchmark. 


\begin{tabular}{|r||r||r||r||r|}
\hline \multicolumn{1}{|r||}{$\mathrm{P}$} & Time & Speedup & Time Vector & Speedup Vector \\
\hline \hline 1 & 62.342 & 1.00 & 49.498 & 1.00 \\
\hline \hline 2 & 31.153 & 2.00 & 24.849 & 1.99 \\
\hline \hline 4 & 15.646 & 3.98 & 12.450 & 3.98 \\
\hline \hline 8 & 7.823 & 7.97 & 6.219 & 7.96 \\
\hline \hline 16 & 3.916 & 15.92 & 3.110 & 15.92 \\
\hline \hline 32 & 1.959 & 31.82 & 1.556 & 31.81 \\
\hline \hline 64 & 0.982 & 63.48 & 0.779 & 63.54 \\
\hline \hline 128 & 0.492 & 126.71 & 0.390 & 126.92 \\
\hline \hline 256 & 0.246 & 253.42 & 0.197 & 251.26 \\
\hline
\end{tabular}

\section{Table 8. FFT Performance on the Cray T3D}

In Table 9 we show the performance of the Cray T3E-600 platform on the FFT benchmark. The execution time and speedup for scalar and vector access to shared memory are shown in the same way as for the T3D. The execution time for a serial implementation of a $2048 \times 2048$ FFT is 16.93 seconds. As was the case for the T3D, vector access to shared memory produces good results on the T3E.

\begin{tabular}{|r||r||r||r||r|}
\hline $\mathrm{P}$ & Time & Speedup & Time Vector & Speedup Vector \\
\hline \hline 1 & 31.66 & 1.00 & 24.11 & 1.00 \\
\hline \hline 2 & 16.26 & 1.95 & 12.16 & 1.98 \\
\hline \hline 4 & 8.36 & 3.79 & 6.08 & 3.96 \\
\hline \hline 8 & 4.33 & 7.31 & 3.05 & 7.91 \\
\hline \hline 16 & 2.19 & 14.46 & 1.52 & 15.88 \\
\hline 32 & 1.12 & 28.25 & 0.76 & 31.72 \\
\hline
\end{tabular}

\section{Table 9. FFT Performance on the Cray T3E-600}

In Table 10 we show the performance of the Meiko CS-2 platform on the FFT benchmark. The execution time for a serial implementation of a $2048 \times 2048$ FFT is 39.96 seconds. The absolute performance and speedup for the FFT benchmark on the 
Meiko CS-2 are poor, caused by the high software overhead placed on shared memory access. Results could be improved through the use of a blocked layout for the 2-D arrays. We will demonstrate this in the results of the matrix multiply benchmark below.

\begin{tabular}{|r||r|r|}
\hline $\mathrm{P}$ & Time & Speedup \\
\hline \hline 1 & 56.76 & 1.00 \\
\hline \hline 2 & 88.70 & 0.64 \\
\hline \hline 4 & 60.77 & 0.93 \\
\hline \hline 8 & 52.99 & 1.07 \\
\hline \hline 16 & 51.07 & 1.11 \\
\hline \hline 32 & 33.07 & 1.72 \\
\hline
\end{tabular}

Table 10. FFT Performance on the Meiko CS-2

\section{Matrix-Matrix Product}

Our matrix multiply benchmark is the computation of the product of two matrices located in shared memory, placing the result in shared memory. This benchmark is for double precision matrices of size $1024 \times 1024$, and is coded in PCP without using vendor-specific libraries to accelerate performance.

In this benchmark we employ a block decomposition for the $1024 \times 1024$ matrices. We treat the matrices as $64 \times 64$ arrays of $16 \times 16$ submatrices. This is done by packing the submatrices into a $\mathrm{C}$ structure. In PCP, shared memory is interleaved on an object boundary where the object in this case is a $\mathrm{C}$ structure. This places the submatrix on one processor and allows the efficient blocked copying of 2048 bytes of memory for each remote memory access.

In the benchmark results below we include for comparison purposes the performance for a serial implementation of the $1024 \times 1024$ matrix multiply, blocked using $16 \times 16$ submatrices. In Tables 11 through 15, we present in columns labeled MFLOPS and Speedup the performance in MFLOPS and the speedup relative to a single processor executing the parallel code, respectively, as a function of the processor count, $\mathrm{P}$.

In Table 11 we show the performance of the DEC 8400 on the matrix multiply benchmark. The performance for a serial implementation of the blocked matrix multiply is 138.41 MFLOPS. Note that the speedups look good up to 4 processors. Our 
implementation of the matrix multiply makes heavier demands upon shared memory bandwidth than the FFT, probably causing the roll off in performance seen here. Note that this was for a system possessing 4 way interleaved memory. Performance may improve if the interleave is 8 or 16 .

\begin{tabular}{|l|r||r|}
\hline $\mathrm{P}$ & MFLOPS & Speedup \\
\hline \hline 1 & 145.06 & 1.00 \\
\hline \hline 2 & 286.37 & 1.97 \\
\hline \hline 4 & 567.84 & 3.91 \\
\hline \hline 8 & 688.47 & 4.75 \\
\hline
\end{tabular}

\section{Table 11. Matrix Multiply Performance on the DEC 8400}

In Table 12 we show the performance of the SGI Origin 2000 on the matrix multiply benchmark. The performance of the serial blocked algorithm on this platform is 126.69 MFLOPS. As was the case for the FFT, the matrix multiply was computed twice and the second pass timed. The virtual memory overhead incurred on the first pass slows down execution by more than a factor of three at thirty processors. The scaling is better than the DEC 8400, but shows signs of diminishing returns above 16 processors.

\begin{tabular}{|r|r||r|}
\hline $\mathrm{P}$ & MFLOPS & Speedup \\
\hline \hline 1 & 109.36 & 1.00 \\
\hline \hline 2 & 213.56 & 1.95 \\
\hline \hline 4 & 407.09 & 3.72 \\
\hline \hline 8 & 777.05 & 7.11 \\
\hline \hline 16 & 1447.45 & 13.24 \\
\hline 20 & 1785.96 & 16.33 \\
\hline \hline 25 & 2192.67 & 20.05 \\
\hline \hline 30 & 2605.40 & 23.82 \\
\hline
\end{tabular}

Table 12. Matrix Multiply Performance on the SGI Origin 2000

In Table 13 we show the performance of the Cray T3D on the matrix multiply 
benchmark. The performance of a serial implementation of the blocked algorithm is 23.38 MFLOPS. We note the superlinear speedups for processor counts between 2 and 8 . This has no explanation in terms of scaling cache size as the processor count is increased. It is likely caused by a performance degradation arising in the use of prefetch logic by a given processor to communicate with its own memory.

\begin{tabular}{|r|r||r|}
\hline $\mathrm{P}$ & MFLOPS & Speedup \\
\hline \hline 1 & 16.20 & 1.00 \\
\hline \hline 2 & 34.38 & 2.12 \\
\hline \hline 4 & 69.34 & 4.28 \\
\hline \hline 8 & 134.49 & 8.30 \\
\hline \hline 16 & 253.48 & 15.65 \\
\hline 32 & 453.79 & 28.01 \\
\hline
\end{tabular}

\section{Table 13. Matrix Multiply Performance on the Cray T3D}

In Table 14 we show the performance of the Cray T3E-600 on the matrix multiply benchmark. The performance of a serial implementation of the blocked algorithm is 97.62 MFLOPS. The T3E, in contrast to the T3D, benefits from an on-chip cache that is fully coherent with the local memory. Memory references from remote processors do not cause gratuitous cache line spills. The parallelization overhead is $24 \%$ and good speedups are obtained.

\begin{tabular}{|r|r|r|}
\hline $\mathrm{P}$ & MFLOPS & Speedup \\
\hline \hline 1 & 78.99 & 1.00 \\
\hline \hline 2 & 158.44 & 2.01 \\
\hline \hline 4 & 314.71 & 3.98 \\
\hline \hline 8 & 624.38 & 7.90 \\
\hline \hline 16 & 1195.12 & 15.13 \\
\hline 32 & 2259.85 & 28.61 \\
\hline
\end{tabular}

Table 14. Matrix Multiply Performance on the Cray T3E-600 
In Table 15 we show the performance of the Meiko CS-2 on the matrix multiply benchmark. The performance of a serial implementation of the blocked algorithm is 14.24 MFLOPS. Note the sharp contrast of the speedups here with the speedups for the case of the FFT benchmark. The blocked data movement has greatly improved performance on the CS-2. Coding for blocked data movement is essential on a distributed memory platform that places high software overhead on communication.

\begin{tabular}{|r|r||r|}
\hline $\mathrm{P}$ & MFLOPS & Speedup \\
\hline \hline 1 & 12.41 & 1.00 \\
\hline \hline 2 & 22.30 & 1.80 \\
\hline \hline 4 & 41.92 & 3.38 \\
\hline \hline 8 & 80.27 & 6.47 \\
\hline 16 & 142.11 & 11.45 \\
\hline 32 & 248.83 & 20.05 \\
\hline
\end{tabular}

\section{Table 15. Matrix Multiply Performance on the Meiko CS-2}

\section{Discussion}

We have investigated the type qualifier approach, first introduced in the Split-C and AC compilers, as an alternative to explicit message passing for writing parallel programs that port across a wide range of architectures. Our investigation was carried out with an extension of the PCP translator that is capable of addressing both shared memory and distributed memory architectures.

The resulting programming model is efficient enough to be the method of choice for shared memory platforms while still providing latency hiding mechanisms that make efficient use of distributed memory architectures possible. Superlinear speedups were obtained for all of the coherent cache shared memory platforms that we tested with the Gaussian elimination benchmark. The inherent scalability of the programming model became clear with the FFT benchmark on the Cray T3D platform, wherein a speedup of 251 for 256 processors was obtained.

A shared memory programming model implemented with the type qualifier approach does not remove the need to exploit latency hiding mechanisms on architectures where communication latency is an obstacle to good performance. Communication latency is significant on all of the distributed memory platforms we tested. Vectorized movement of 
data between shared and private memory was found to be sufficient to provide good performance on the Cray T3D and T3E platforms. It was refreshing to see true distributed memory systems provide good performance when using well known and understood techniques that did not require much code restructuring.

The Meiko CS-2, however, does not possess communication hardware that is capable of efficiently overlapping vector access to arrays of single words. More aggressive code restructuring to provide large block access to shared data is required in order to obtain good performance. We demonstrated the value of blocking shared data access on the Meiko CS-2 in the matrix multiply benchmark.

\section{Acknowledgments}

* Work performed under the auspices of the U.S. Department of Energy by the Lawrence Livermore National Laboratory under contract No. W-7405-ENG-48.

${ }^{1}$ This work was partially supported by National Center for Supercomputing Applications under grant number CCR970005N and utilized the Silicon Graphics Origin 2000 at the National Center for Supercomputing Applications, University of Illinois at Urbana-Champaign.

2 This research used resources of the National Energy Research Scientific Computing Center, which is supported by the Office of Energy Research of the U.S. Department of Energy.

\section{References}

[1] MPI: A Message-Passing Interface Standard, Message Passing Interface Forum, Version 1.1, University of Tennessee, Knoxville, Tennessee, June 1995.

[2] David E. Culler, et al, Parallel Programming in Split-C, Proc. Supercomputing 93, IEEE, pp. 262-273, Nov. 1993.

[3] W. W. Carlson and J. M. Draper, Distributed Data Access in AC, SIGPLAN Notices, vol 30, number 8, pp. 39-47, July 1995.

[4] T. von Eicken, D. E. Culler, S. C. Goldstein, K. E. Schauser, Active Messages: a Mechanism for Integrated Communication and Computation, Proceedings of the 
International Symposium on Computer Architecture, 1992.

[5] E. D. Brooks III, K. H. Warren, A Parallel Programming Model Bridging Shared Memory and Distributed Memory Architectures, UCRL-JC-126451, Lawrence Livermore National Laboratory, January, 1997.

[6] E. D. Brooks III, B. C. Gorda, K. H. Warren, The Parallel C Preprocessor, Scientific Programming, vol. 1, no.1, pp 79-89, Fall 1992.

[7] AlphaServer 8200/8400 Systems: Technical Summary, Digital Equipment Corporation, 1996.

[8] Sarita V. Adve and Kourosh Charachorloo, Shared Memory Consistency Models: A Tutorial, WRL Research Report 95/7, Digital Western Research Laboratory, Palo Alto, CA 94301, September 1995.

[9] Technical Overview of the Origin Family, Silicon Graphics Inc., 1997.

[10] Leslie Lamport, How to Make a Multiprocessor Computer that Correctly Executes Multiprocess Programs, IEEE Transactions on Computers, C-28(9):690-691, September 1979.

[11] Communications Processor Overview, S1002-10M100, Meiko World Incorporated, 1993.

[12] L. Lamport, A Fast Mutual Exclusion Algorithm, ACM Trans. Comput. Syst. 15, 1, 1-11, Feb. 1987.

[13] Elan Widget Library, S1002-10M104.07, Meiko World Incorporated, 1993.

[14] W.H. Press et al, Numerical Recipes in C, Second Edition, pp 41-42, Cambridge University Press, 1995.

[15] B. Gorda, R. Wolski, Time Sharing Massively Parallel Machines, Proc. 1995 International Conference on Parallel Processing, pp. II-214 to II-217, August, 1995.

[16] W.H. Press et al, Numerical Recipes in C, Second Edition, pp 507-508, Cambridge University Press, 1995. 


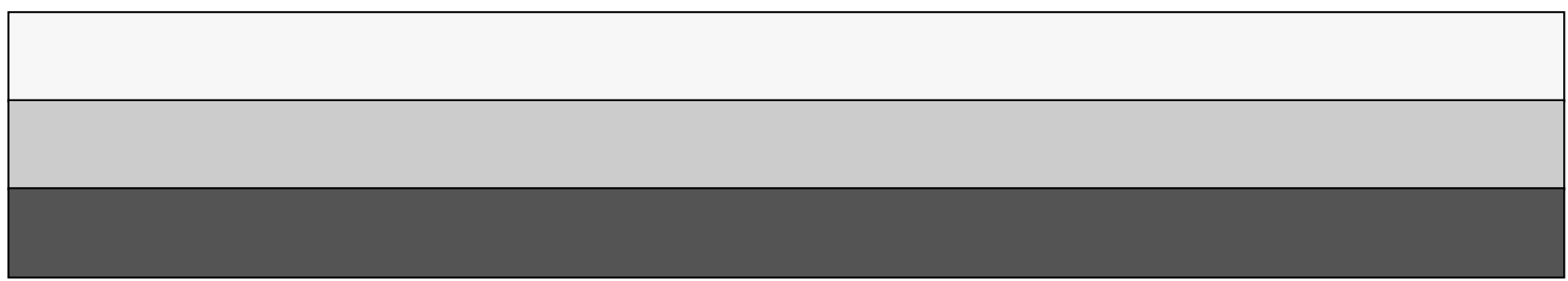

